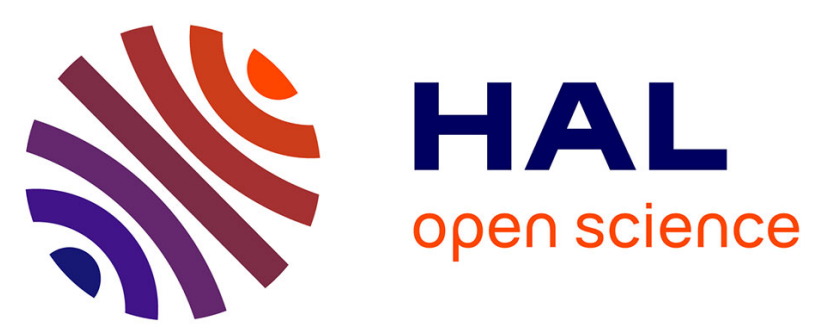

\title{
Design of Balanced QPSK Space-Time Trellis Codes for Several Transmit Antennas
}

Thi Minh Hien Ngo, Gheorghe Zaharia, Stéphane Bougeard, Jean-François Hélard

\section{- To cite this version:}

Thi Minh Hien Ngo, Gheorghe Zaharia, Stéphane Bougeard, Jean-François Hélard. Design of Balanced QPSK Space-Time Trellis Codes for Several Transmit Antennas. International Symposium on Signals, Circuits and Systems, 2007. ISSCS 2007., Jul 2007, Romania. pp.1-4. hal-00172738

\section{HAL Id: hal-00172738 \\ https://hal.science/hal-00172738}

Submitted on 17 Sep 2007

HAL is a multi-disciplinary open access archive for the deposit and dissemination of scientific research documents, whether they are published or not. The documents may come from teaching and research institutions in France or abroad, or from public or private research centers.
L'archive ouverte pluridisciplinaire HAL, est destinée au dépôt et à la diffusion de documents scientifiques de niveau recherche, publiés ou non, émanant des établissements d'enseignement et de recherche français ou étrangers, des laboratoires publics ou privés. 


\title{
Design of Balanced QPSK Space-Time Trellis Codes for Several Transmit Antennas
}

\author{
Thi Minh Hien Ngo, Gheorghe Zaharia, Stéphane Bougeard, Jean François Hélard \\ Institute for Electronics and Telecommunications of Rennes (IETR) - UMR CNRS 6164 \\ INSA - 20 avenue des Buttes de Coësmes, 35043 Rennes, France \\ Email: Minh-Hien.Ngothi@ens.insa-rennes.fr
}

\begin{abstract}
A new class of QPSK Space Time Trellis Codes (STTC) for several transmit antennas is proposed in this paper. We call these codes Balanced STTC because they use the points of the constellation with the same probability. Comparing to known codes, these codes offer the best performance. Therefore, the systematic search for good codes can be reduced to this class. It is shown that all the best published codes are balanced. The paper presents the design of these balanced STTC and gives a complete list of the best 4 -state codes for 2 transmit antennas. Several 16-state balanced codes for 2 and 3 transmit antennas are also given.
\end{abstract}

\section{INTRODUCTION}

Space Time Trellis Coded Modulation (STTCM) was introduced in 1998 by Tarokh et al. [1] by combining channel coding with the Multiple Input Multiple Output (MIMO) concept to improve the data rate and the reliability of wireless communications. Many performance criteria have been established to maximize both diversity and coding gain of STTC. The rank and determinant criteria for slow fading channels with the Euclidian distance and the product distance criteria for fast fading channels have been proposed in [1]. In [2] Chen introduced the trace criterion which governs the coding for systems with a great product of the numbers of transmit $(\mathrm{Tx})$ and receive $(\mathrm{Rx})$ antennas.

Based on above criteria, many different STTC for $2 \mathrm{Tx}$ antennas have been found by a systematic code search [3][6]. The performance study of these codes was carried out over slow and fast Rayleigh fading channels to identify the most efficient ones [7]. It has been shown that over slow fading channels, the codes constructed with the trace criterion give similar or even better results that the codes constructed with the rank and the determinant criteria. Over fast fading channels, "trace criterion codes" as Chen's codes outperform the other tested codes. In the same way, some codes for $3 \mathrm{Tx}$ antennas have been published in [6], [8]. All the codes which achieve the best performance have the same property: they use the points of the constellation with the same probability if the data are generated by a binary memoryless source with equally probable symbols. Therefore, we call these codes "BalancedSTTC" (B-STTC) [9].

Until now, no efficient construction method of STTC has been proposed and systematic code search has been employed to obtain STTC with good performance [3]-[6]. The main contribution of this paper is the description of a method of construction of this new class of codes which offers the best performance. Therefore, the systematic search for good codes can be reduced to this class. A table with all the best 4state STTC and a table with some best 16-state STTC for 2 Tx antennas are given. Some best 16-state STTC for 3 Tx antennas are also presented. Finally, the performance of all these codes is evaluated by simulation.

The rest of the paper is organized as follows. Section II briefly describes the STTC. The new class of balanced codes is introduced in Section III and their properties are listed in Section IV. The design of QPSK balanced space-time trellis codes for several Tx antennas is described in Section $\mathrm{V}$ and VI respectively. Finally, it is shown in Section VII that the best B-STTC outperform or equal previously known codes.

\section{SPACE TIME TRELLIS CODING}

We consider the general case of $2^{n}$-PSK space-time trellis encoder as shown on Fig.1 ( $n=2$ for QPSK modulation).
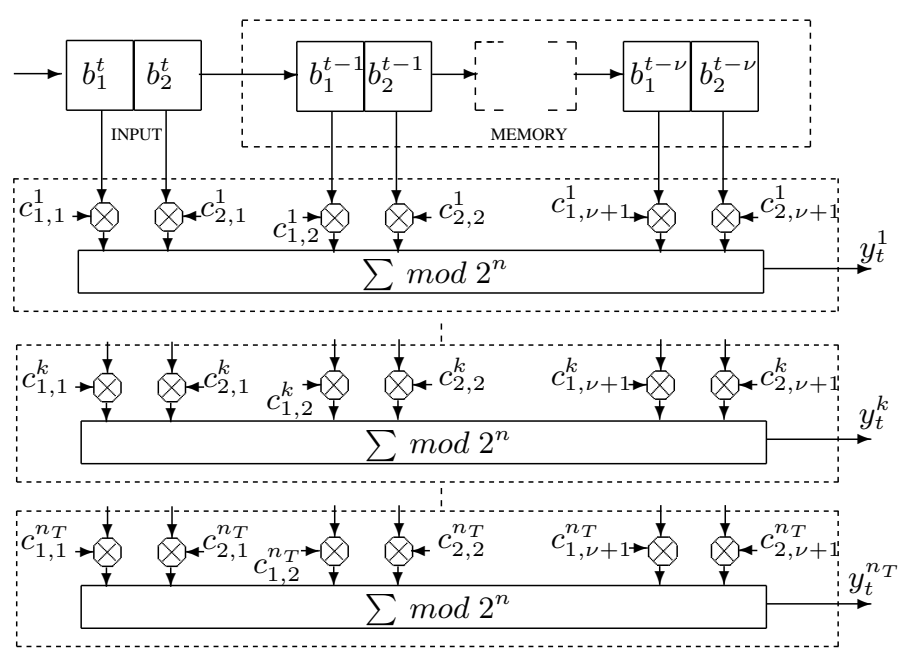

Fig. 1. ST trellis encoder for $2^{n}$-PSK and $n_{T}$ Tx antennas

This encoder is composed of one input block of $n$ bits and $\nu$ memory blocks of $n$ bits. At each time $t \in \mathbb{Z}$, all the bits of a block are replaced by the $n$ bits of the previous block. The $i^{t h}$ bit $b_{i}^{t-j+1}, i=1 \ldots n$, of the $j^{t h}$ block, $j=1 \ldots \nu+1$, is associated to $n_{T}$ multiplier coefficients $c_{i, j}^{k} \in \mathbb{Z}_{2^{n}}, k=1 \ldots n_{T}$ where $n_{T}$ is the number of Tx antennas. A ST trellis encoder is thus classically defined by 
its generator matrix $\mathbf{C}$ of $n_{T} \times n(\nu+1)$ coefficients:

$$
\mathbf{C}=\left[\begin{array}{ccccccc}
c_{1,1}^{1} & \ldots & c_{n, 1}^{1} & \ldots & c_{1, \nu+1}^{1} & \ldots & c_{n, \nu+1}^{1} \\
\vdots & & & \ldots & & & \vdots \\
c_{1,1}^{k} & \ldots & c_{n, 1}^{k} & \ldots & c_{1, \nu+1}^{k} & \ldots & c_{n, \nu+1}^{k} \\
\vdots & & & \ldots & & & \vdots \\
c_{1,1}^{n_{T}} & \ldots & c_{n, 1}^{n_{T}} & \ldots & c_{1, \nu+1}^{n_{T}} & \ldots & c_{n, \nu+1}^{n_{T}}
\end{array}\right]
$$

\section{BALANCED CODES}

\section{A. Why balanced codes?}

The concept of "balanced codes" is based on the observation that all the good STTC proposed in the literature present the same property: the generated symbols of the constellation are equally probable.

Indeed, if the binary input data is generated by a memoryless source $\mathbf{S}=\{0,1\}$ with equally probable symbols, then, in the case of 4 -PSK modulation, from a given state $\mathbf{X}=\left[x_{1} x_{2} \ldots x_{L}\right]^{T} \in \mathbb{Z}_{2}^{L}$ of the shift-register realized by $(\nu+1)$ blocks of $n$ bits, the MIMO symbol $\mathbf{Y}=\left[y_{1} y_{2} \ldots y_{n_{T}}\right]^{T} \in \mathbb{Z}_{4}^{n_{T}}$ generated by the STTC encoder shown in Fig.1 is:

$$
\mathbf{Y}=\mathbf{C} \cdot \mathbf{X}
$$

where $\mathbf{C}$ is the generator matrix (1). This is a deterministic relation. Therefore, the STTC is defined by a map:

$$
\boldsymbol{\Phi}: \mathbb{Z}_{2}^{L} \rightarrow \mathbb{Z}_{4}^{n_{T}}
$$

which associates to the state $\mathbf{X}$ an unique codeword $\mathbf{Y}$. Note that $\boldsymbol{\Phi}\left(\mathbb{Z}_{2}^{L}\right) \subseteq \mathbb{Z}_{4}^{n_{T}}$ represents the set of generated codewords $\mathbf{Y}$. A given codeword $\mathbf{Y}$ can be obtained for several states $\mathbf{X} \in \mathbb{Z}_{2}^{L}$. Let $n(\mathbf{Y})$ be the number of occurrences of the codeword $\mathbf{Y}$.

By definition, a STTC is balanced if and only if each generated codeword $\mathbf{Y} \in \mathbf{\Phi}\left(\mathbb{Z}_{2}^{L}\right)$ has the same number of occurrences $n(\mathbf{Y})=n_{0} \geq 1$.

In addition, if $\boldsymbol{\Phi}\left(\mathbb{Z}_{2}^{L}\right)=\mathbb{Z}_{4}^{n_{T}}$, then all the codewords are generated and the STTC is fully balanced.

Due to the random source $\mathbf{S}=\{0,1\}$, from a given state $\mathbf{X}$ the encoder can have only 4 equally probable next states. The matrix $\mathbf{T}$ of the transition probabilities between these states corresponds to a Markov chain. Due to the symmetry of the matrix $\mathbf{T}$, the steady state probabilities of the states $\mathbf{X}$ are all equal. For a balanced code, by using (2), the generated codewords $\mathrm{Y}$ are also equally probable. In other words, the generated symbols of the constellation are equally probable.

\section{B. Properties of balanced STTC}

The design of the B-STTC is based on the following properties:

Theorem 1 If a MIMO code with a $L$-length shift-register is fully balanced then $L \geq L_{\text {min }}=n \cdot n_{T}=\operatorname{dim}\left(\mathbb{Z}_{2^{n}}^{n_{T}}\right)$.

Theorem 2 Let us consider a balanced MIMO code with a $L$-length shift-register. Then, for any additional column matrix $\mathbf{C}_{i} \in \mathbb{Z}_{2^{n}}^{n_{T}}$, the resulting MIMO code with a $(L+1)$-length shift-register is also balanced.
Definition 1 The vectors $\mathbf{C}_{1}, \mathbf{C}_{2}, \ldots, \mathbf{C}_{L}$ are linearly independent if the equation

$$
x_{1} \mathbf{C}_{1}+x_{2} \mathbf{C}_{2}+\cdots+x_{L} \mathbf{C}_{L}=\mathbf{0} \in \mathbb{Z}_{2^{n}}^{n_{T}}
$$

with $x_{i} \in\{0,1\}$ holds if and only if all $x_{i}=0$.

Definition 2 A set of linearly independent vectors $\mathbf{C}_{1}, \ldots, \mathbf{C}_{m}$ is called a base for $\mathbb{Z}_{2^{n}}^{n_{T}}$ if and only if

$$
\operatorname{span}\left(\mathbf{C}_{1}, \mathbf{C}_{2}, \ldots, \mathbf{C}_{m}\right)=\left\{\sum_{i=1}^{m} x_{i} \mathbf{C}_{i} / x_{i} \in\{0,1\}\right\}=\mathbb{Z}_{2^{n}}^{n_{T}} .
$$

In this case, $m=n . n_{T}$ is the dimension of the set $\mathbb{Z}_{2^{n}}^{n_{T}}$.

\section{PRoperties of the BASES of $\mathbb{Z}_{4}^{n_{T}}$}

In the following, a method to design fully balanced codes is presented. The properties of the bases of $\mathbb{Z}_{4}^{n_{T}}$, which characterize the fully balanced codes are listed. Further, the systematic search will be performed only for these fully balanced codes.

Property 1: The null vector $0 \in \mathbb{Z}_{4}^{n_{T}}$ can not be used to form a base.

Property 2: If the vector $\mathbf{C}_{i} \in \mathbb{Z}_{4}^{n_{T}}$ is used to form a base, then $\mathbf{C}_{j}=-\mathbf{C}_{i}$ does not belong to this base.

Property 3: If the vectors $\mathbf{C}_{1}, \mathbf{C}_{2}, \ldots, \mathbf{C}_{m} \in \mathbb{Z}_{4}^{n_{T}}$ with $m<L_{\min }=n . n_{T}$ are linearly independent, then the vectors

$$
\mathbf{C}_{m+1}=\sum_{i=1}^{m} \lambda_{i} \mathbf{C}_{i} \in \mathbb{Z}_{4}^{n_{T}}, \quad \lambda_{i} \in\{-1,0,1\}
$$

can not be used to obtain $m+1$ linearly independent vectors.

Property 4: $\mathcal{C}_{0}=2 \mathbb{Z}_{2}^{n_{T}}$ is a normal subgroup of the additive group $\mathbb{Z}_{4}^{n_{T}}$. For each element $v \in \mathbb{Z}_{2}^{n_{T}}$ we consider the coset $\mathcal{C}_{v}=v+2 \mathbb{Z}_{2}^{n_{T}}$, where addition is in $\mathbb{Z}_{4}^{n_{T}}$.

Property 5: If $u_{1} \in \mathcal{C}_{u}$ and $v_{1} \in \mathcal{C}_{v}$ then $u_{1}+v_{1} \in \mathcal{C}_{u \oplus v}$, where $\oplus$ represents the addition in $\mathbb{Z}_{2}^{n_{T}}$.

Property 6: If $u_{1} \in \mathcal{C}_{u}$ then $u_{1}+\mathcal{C}_{v}=\mathcal{C}_{u \oplus v}$.

Property 7: $u+\mathcal{C}_{u}=\mathcal{C}_{0}=2 \mathbb{Z}_{2}^{n_{T}}$.

Property 8: The sum of two cosets is defined by

$$
\mathcal{C}_{u}+\mathcal{C}_{v}=\left\{u_{1}+v_{1} / u_{1} \in \mathcal{C}_{u} \text { and } v_{1} \in \mathcal{C}_{v}\right\}
$$

Property 9: The direct sum of two cosets is a coset:

$$
\mathcal{C}_{u}+\mathcal{C}_{v}=\mathcal{C}_{u \oplus v}
$$

Property 10: If $u_{1} \in \mathcal{C}_{u}$ then $-u_{1} \in \mathcal{C}_{u}$.

Property 11: If $p_{1}, p_{2}, \ldots, p_{n_{T}} \in \mathbb{Z}_{2}^{n_{T}} \backslash\{0\}$ are linearly independent then $\operatorname{span}\left(2 p_{1}, 2 p_{2}, \ldots, 2 p_{n_{T}}\right)=2 \mathbb{Z}_{2}^{n_{T}}=\mathcal{C}_{0}$.

Property 12: If $p \in \mathbb{Z}_{2}^{n_{T}}$ then the sum of 2 different elements of the coset $\mathcal{C}_{p}$ is an element of $\mathcal{C}_{0} \backslash\{2 p\}$.

Property 13: One base of $\mathbb{Z}_{4}^{n_{T}}$ contains at least one vector in $\mathcal{C}_{0}$.

Property 14: One base of $\mathbb{Z}_{4}^{n_{T}}$ contains at most $n_{T}$ vectors in the same coset.

Property 15: One base of $\mathbb{Z}_{4}^{n_{T}}$ always contains $n_{T}$ vectors which belong to $n_{T}$ cosets $\mathcal{C}_{p_{1}}, \mathcal{C}_{p_{2}}, \ldots, \mathcal{C}_{p_{n_{T}}}$ different from $\mathcal{C}_{0}$ such as the vectors $p_{1}, p_{2}, \ldots, p_{n_{T}}$ are linearly independent. Similarly, the cosets $\mathcal{C}_{p_{1}}, \mathcal{C}_{p_{2}}, \ldots, \mathcal{C}_{p_{n_{T}}}$ are called linearly independent. 


\section{DESIGN OF 4-PSK STTC With 2 TX ANTENNAS}

The design of the fully balanced codes includes 2 steps:

- First step: generation of all the bases of $\mathbb{Z}_{4}^{2}$.

- Second step: permutation of the column vectors of each obtained base to generate all the fully balanced codes.

Table I shows the partition of $\mathbb{Z}_{4}^{2}$ in 4 cosets.

TABLE I. Partition of $\mathbb{Z}_{4}^{2}$ in cosets

\begin{tabular}{|c|c|c|c|c|}
\hline $\mathcal{C}_{[0]}$ & 0 & 0 & 2 & 2 \\
\hline$\left[\begin{array}{l}0 \\
0 \\
0\end{array}\right]$ & 0 & 2 & 0 & 2 \\
\hline $\mathcal{C}_{[0]}$ & 0 & $\begin{array}{l}0 \\
3\end{array}$ & 2 & 2 \\
\hline & 1 & 1 & 3 & 3 \\
\hline $\mathcal{C}^{\mathcal{C}}\left[\begin{array}{l}1 \\
0\end{array}\right]$ & 0 & 2 & 0 & 2 \\
\hline $\mathcal{C}_{[1]}$ & $\begin{array}{l}1 \\
1\end{array}$ & $\begin{array}{l}1 \\
3\end{array}$ & $\begin{array}{l}3 \\
1\end{array}$ & $\begin{array}{l}3 \\
3\end{array}$ \\
\hline
\end{tabular}

There are two types of fully balanced codes of $\mathbb{Z}_{4}^{2}$ :

- Type $I$ codes which contain only 1 non-null vector in $\mathcal{C}_{0}$

- Type $I I$ codes which contain 2 non-null vectors in $\mathcal{C}_{0}$

\section{A. Design of fully balanced codes of type I}

The algorithm to obtain a base of type I is as follows:

1) Choose one vector $2 p \in \mathcal{C}_{0}^{*}=\mathcal{C}_{0} \backslash\{0\}$.

2) Choose 2 linearly independent vectors in $\mathcal{C}_{p}$.

3) Choose the last vector in a different coset.

There are 96 bases of type $I$ in $\mathbb{Z}_{4}^{2}$ [9].

\section{B. Design of fully balanced codes of type II}

The algorithm to obtain a base of type II is as follows:

1) Choose 2 different vectors in $\mathcal{C}_{0}^{*}$.

2) Choose 2 different cosets $\mathcal{C}_{u}$ and $\mathcal{C}_{v}$ different from $\mathcal{C}_{0}$.

3) For each of these 2 cosets, choose one representative.

There are 144 bases of type $I I$ in $\mathbb{Z}_{4}^{2}$ [9].

The total number of the bases of $\mathbb{Z}_{4}^{2}$ is: $96+144=240$ bases.

\section{DESIGn of 4-PSK STTC With 3 Tx Antennas}

Similarly to the design of 4-PSK STTC with 2 Tx antennas, this section is focused on the design of bases of $\mathbb{Z}_{4}^{3}$.

One base of $\mathbb{Z}_{4}^{3}$ contains at least one vector in $\mathcal{C}_{0}$ and at most 3 vectors in $\mathcal{C}_{0}$ (Properties 13, 14). Then, there are three types of fully balanced codes of $\mathbb{Z}_{4}^{3}$ :

- Type $I$ codes which contain only 1 non-null vector in $\mathcal{C}_{0}$

- Type $I I$ codes which contain 2 non-null vectors in $\mathcal{C}_{0}$

- Type $I I I$ codes which contain 3 non-null vectors in $\mathcal{C}_{0}$

Design of fully balanced codes of type III

The algorithm to obtain a type $I I I$ base of $\mathbb{Z}_{4}^{3}$ is as follows:

1) Choose 3 linearly independent vectors of $\mathcal{C}_{0}$.

2) Choose 3 linearly independent vectors $u, v, w \in \mathbb{Z}_{2}^{3}$. Therefore, the cosets $\mathcal{C}_{u}, \mathcal{C}_{v}, \mathcal{C}_{w} \in \mathbb{Z}_{4}^{3}$ are linearly independent.

3) Choose the vectors $u_{1} \in \mathcal{C}_{u}, v_{1} \in \mathcal{C}_{v}, w_{1} \in \mathcal{C}_{w}$. Because $u_{1}+\mathcal{C}_{0}=\mathcal{C}_{u}$, the whole coset $\mathcal{C}_{u}$ is generated. In the similar way, $\mathcal{C}_{v}$ and $\mathcal{C}_{w}$ are generated. By using Property 9 , the other cosets are also generated.
Finally, the total sum of the bases for the fully balanced codes of type $I I I$ is 401408 bases.

In a similar way, we obtain 946176 bases for the fully balanced codes of type $I I$ and 516096 bases for the fully balanced codes of type $I$. Finally, the total number of the bases in $\mathbb{Z}_{4}^{3}$ is: $401408+946176+516096=1863680$ bases.

In the general case of $\mathbb{Z}_{4}^{n_{T}}$ there are $n_{T}$ types fully balanced codes. There are $N_{B}=\frac{1}{n_{T} !} \prod_{k=0}^{n_{T}-1}\left(2^{n_{T}}-2^{k}\right)$ bases of $\mathbb{Z}_{2}^{n_{T}}$ and $N_{B}^{2} * 2^{n_{T}^{2}}$ bases of type $n_{T}$ of $\mathbb{Z}_{4}^{n_{T}}$.

Because $\operatorname{dim}\left(\mathbb{Z}_{4}^{n_{T}}\right)=2 n_{T}$, each permutation of $2 n_{T}$ vectors forming a base of $\mathbb{Z}_{4}^{n_{T}}$ generates a different balanced code. Therefore, there are $N_{C}=2^{n_{T}^{2}} *\left(2 n_{T}\right) ! * N_{B}^{2}$ balanced STTC of type $n_{T}$.

\section{CODE PERFORMANCE}

Before showing all the best codes based on the trace criterion, we propose herein some trace properties of 4-state 4-PSK STTC:

- Property $P_{1}$ : the codes $\mathbf{C}=\left[\begin{array}{lll}\mathbf{C}_{1} \mathbf{C}_{2} & \mathbf{C}_{3} \mathbf{C}_{4}\end{array}\right]$, $\mathbf{C}^{\prime}=\left[\begin{array}{lll}-\mathbf{C}_{1} \mathbf{C}_{2} & \mathbf{C}_{3} \mathbf{C}_{4}\end{array}\right]$ and $\mathbf{C}^{\prime \prime}=\left[\begin{array}{lll}\mathbf{C}_{1} \mathbf{C}_{2} & -\mathbf{C}_{3} \mathbf{C}_{4}\end{array}\right]$ have the same minimum trace.

- Property $P_{2}$ : the codes $\mathbf{C}=\left[\begin{array}{lll}\mathbf{C}_{1} \mathbf{C}_{2} & \mathbf{C}_{3} \mathbf{C}_{4}\end{array}\right]$ and $\mathbf{C}^{\prime}=\left[\begin{array}{lll}\mathbf{C}_{2} \mathbf{C}_{1} & \mathbf{C}_{3} \mathbf{C}_{4}\end{array}\right]$ have the same minimum trace.

- Property $P_{3}$ : the codes $\mathbf{C}=\left[\begin{array}{lll}\mathbf{C}_{1} \mathbf{C}_{2} & \mathbf{C}_{3} \mathbf{C}_{4}\end{array}\right]$ and $\mathbf{C}^{\prime}=\left[\begin{array}{lll}\mathbf{C}_{3} \mathbf{C}_{4} & \mathbf{C}_{1} \mathbf{C}_{2}\end{array}\right]$ achieve the same minimum trace.

- Property $P_{4}$ : the same minimum trace is obtained by using a permutation between the rows of the generator matrix C, i.e, a permutation between the indices of the Tx antennas.

An exhaustive computer search is carried out to detect all the 4-state 4-PSK STTC with 2 Tx antennas that achieve the maximum rank and the maximum trace. A set of 80 codes with $\min (\operatorname{rank}(\mathbf{A}))=2$ and $\min (\operatorname{tr}(\mathbf{A}))=10$ is found. All these codes offer a minimum product distance $d_{p}^{2}=4 \cdot 6=24$ which is the best product distance that can be achieved for 4-state 4-PSK STTC with 2 Tx antennas.

Table II contains all the 4-state fully balanced codes of type II which offer the best performance over fast and slow Rayleigh fading channels with two or more Rx antennas. There are not other codes with better performance than the codes given in this table.

TABLE II. 4-state 4-PSK fully B-STTC with 2 Tx antennas and $\min (\operatorname{tr}(\mathbf{A}))=10$

\begin{tabular}{|c|c|c|c|c|c|c|c|c|c|c|c|c|c|c|c|}
\hline$\left[\begin{array}{l}1 \\
2\end{array}\right.$ & $\begin{array}{l}2 \\
0\end{array}$ & $\begin{array}{l}0 \\
2\end{array}$ & $\left.\begin{array}{l}2 \\
1\end{array}\right]$ & {$\left[\begin{array}{l}1 \\
2\end{array}\right.$} & $\begin{array}{l}2 \\
0\end{array}$ & $\begin{array}{l}0 \\
2\end{array}$ & $\left.\begin{array}{l}2 \\
3\end{array}\right]$ & {$\left[\begin{array}{l}3 \\
2\end{array}\right.$} & $\begin{array}{l}2 \\
0\end{array}$ & $\begin{array}{l}0 \\
2\end{array}$ & $\left.\begin{array}{l}2 \\
1\end{array}\right]$ & {$\left[\begin{array}{l}3 \\
2\end{array}\right.$} & $\begin{array}{l}2 \\
0\end{array}$ & $\begin{array}{l}0 \\
2\end{array}$ & $\left.\begin{array}{l}2 \\
3\end{array}\right]$ \\
\hline$\left[\begin{array}{l}2 \\
0\end{array}\right.$ & $\begin{array}{l}1 \\
2\end{array}$ & $\begin{array}{l}2 \\
1\end{array}$ & $\left.\begin{array}{l}0 \\
2\end{array}\right]$ & {$\left[\begin{array}{l}2 \\
0\end{array}\right.$} & $\begin{array}{l}1 \\
2\end{array}$ & $\begin{array}{l}2 \\
3\end{array}$ & $\left.\begin{array}{l}0 \\
2\end{array}\right]$ & {$\left[\begin{array}{l}2 \\
0\end{array}\right.$} & $\begin{array}{l}3 \\
2\end{array}$ & $\begin{array}{l}2 \\
1\end{array}$ & $\left.\begin{array}{l}0 \\
2\end{array}\right]$ & {$\left[\begin{array}{l}2 \\
0\end{array}\right.$} & $\begin{array}{l}3 \\
2\end{array}$ & $\begin{array}{l}2 \\
3\end{array}$ & $\left.\begin{array}{l}0 \\
2\end{array}\right]$ \\
\hline$\left[\begin{array}{l}2 \\
1\end{array}\right.$ & $\begin{array}{l}0 \\
2\end{array}$ & $\begin{array}{l}2 \\
0\end{array}$ & $\left.\begin{array}{l}1 \\
2\end{array}\right]$ & {$\left[\begin{array}{l}2 \\
1\end{array}\right.$} & $\begin{array}{l}0 \\
2\end{array}$ & $\begin{array}{l}2 \\
0\end{array}$ & $\left.\begin{array}{l}3 \\
2\end{array}\right]$ & {$\left[\begin{array}{l}2 \\
3\end{array}\right.$} & $\begin{array}{l}0 \\
2\end{array}$ & $\begin{array}{l}2 \\
0\end{array}$ & $\left.\begin{array}{l}1 \\
2\end{array}\right]$ & {$\left[\begin{array}{l}2 \\
3\end{array}\right.$} & $\begin{array}{l}0 \\
2\end{array}$ & $\begin{array}{l}2 \\
0\end{array}$ & $\left.\begin{array}{l}3 \\
2\end{array}\right]$ \\
\hline$\left[\begin{array}{l}0 \\
2\end{array}\right.$ & $\begin{array}{l}2 \\
1\end{array}$ & $\begin{array}{l}1 \\
2\end{array}$ & $\left.\begin{array}{l}2 \\
0\end{array}\right]$ & {$\left[\begin{array}{l}0 \\
2\end{array}\right.$} & $\begin{array}{l}2 \\
1\end{array}$ & $\begin{array}{l}3 \\
2\end{array}$ & $\left.\begin{array}{l}2 \\
0\end{array}\right]$ & {$\left[\begin{array}{l}0 \\
2\end{array}\right.$} & $\begin{array}{l}2 \\
3\end{array}$ & $\begin{array}{l}1 \\
2\end{array}$ & $\left.\begin{array}{l}2 \\
0\end{array}\right]$ & {$\left[\begin{array}{l}0 \\
2\end{array}\right.$} & $\begin{array}{l}2 \\
3\end{array}$ & $\begin{array}{l}3 \\
2\end{array}$ & $\left.\begin{array}{l}2 \\
0\end{array}\right]$ \\
\hline
\end{tabular}

In this table, all the codes are related due to the trace properties presented before.

For the 16-state 4-PSK STTC, the construction and the research of the best codes are made from the 4-state 4-PSK 
STTC by using Theorem 2. Table III contains all the 16-state B-STTC which offer the best performance over fast and slow Rayleigh fading channels with two or more Rx antennas. All these codes have $\min (\operatorname{tr}(\mathbf{A}))=16$ and offer a minimum product distance $d_{p}^{2}=128$. Among them, we found the code proposed by Chen $\left[\begin{array}{llllll}1 & 2 & 1 & 2 & 3 & 2 \\ 2 & 0 & 3 & 2 & 2 & 0\end{array}\right]$ (in bold in Tab. III).

TABLE III. 16-state 4-PSK fully B-STTC with 2 Tx antennas and $\min (\operatorname{tr}(\mathbf{A}))=16$

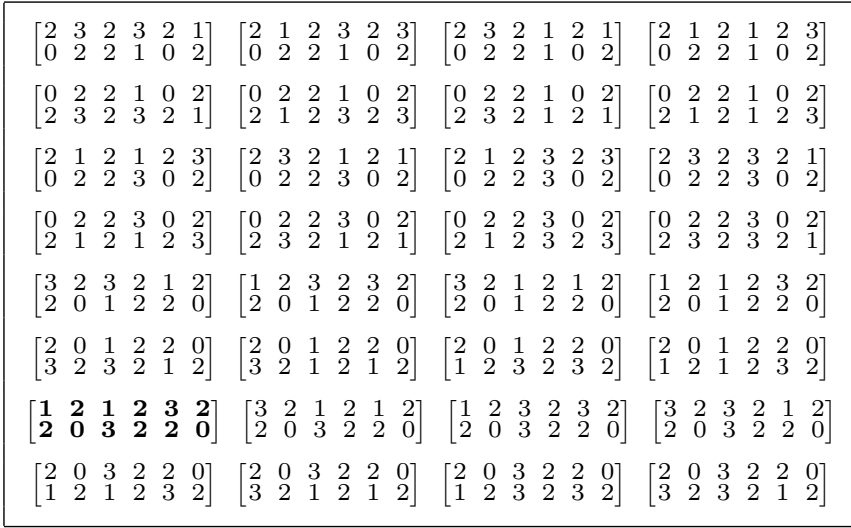

Finally, the performance of all these 4-state and 16-state codes is evaluated by simulation and described by the Frame Error Rate (FER) and Bit Error Rate (BER) over fast Rayleigh fading channels. The results are showed in Fig. 2. Note that all the codes given in Tab. II achieve the same performance than the code of Chen [2] and they offer better performance than the codes proposed in [5].

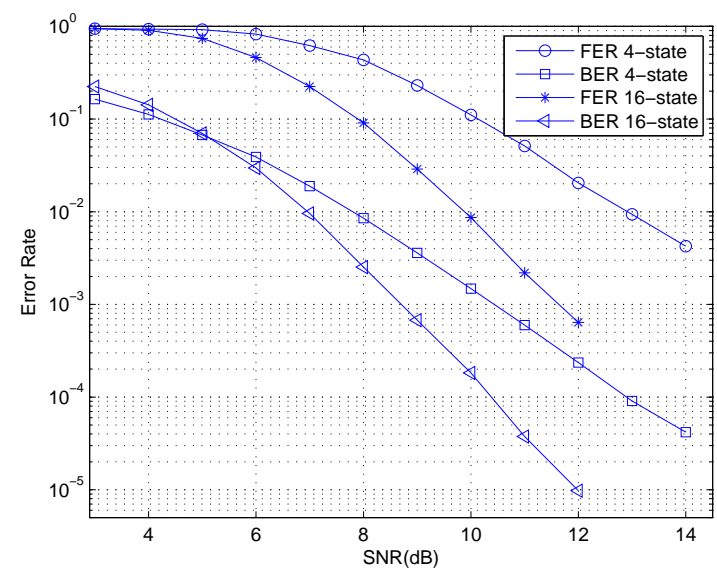

Fig. 2. Performance of B-STTC 4-PSK with 2 Tx and 2 Rx antennas over fast Rayleigh fading channels, frame length 128 symbols

For the 16-state 4-PSK with 3 antennas, after having analyzed the fully balanced codes of type $I I I$, a set of 7296 codes with $\min (\operatorname{rank}(\mathbf{A}))=3$ and $\min (\operatorname{tr}(\mathbf{A}))=24$ is found. All of them achieve the same trace than $\left[\begin{array}{llllll}1 & 2 & 1 & 2 & 3 & 2 \\ 2 & 0 & 3 & 2 & 2 & 0 \\ 1 & 2 & 2 & 0 & 1 & 2\end{array}\right]$ given in [8] and $\left[\begin{array}{llllll}1 & 1 & 3 & 2 & 2 & 2 \\ 2 & 3 & 2 & 0 & 2 & 0 \\ 3 & 2 & 3 & 2 & 0 & 2\end{array}\right]$ proposed in [10]. Some of these 7296 codes are represented in Tab.IV. Similarly to the case with $2 \mathrm{Tx}$ antennas, by using the trace properties for 3 Tx antennas, we can also generate the other codes with the same performance from each code in Tab.IV.

TABLE IV. 16-state 4-PSK fully balanced STTC with 3 Tx antennas and $\min (\operatorname{tr}(\mathbf{A}))=24$

\begin{tabular}{|c|c|c|c|}
\hline$\left[\begin{array}{llllll}0 & 2 & 2 & 0 & 2 & 3\end{array}\right]$ & {$\left[\begin{array}{llllll}2 & 0 & 2 & 3 & 0 & 2\end{array}\right]$} & {$\left[\begin{array}{llllll}0 & 2 & 2 & 3 & 0 & 2\end{array}\right]$} & {$\left[\begin{array}{llllll}0 & 2 & 0 & 0 & 0 & 1\end{array}\right]$} \\
\hline $\begin{array}{llllll}0 & 2 & 2 & 2 & 3 & 2\end{array}$ & $\begin{array}{llllll}0 & 2 & 2 & 2 & 1 & 2\end{array}$ & $\begin{array}{llllll}3 & 2 & 2 & 3 & 2 & 0\end{array} \mid$ & $\begin{array}{lllllll}0 & 2 & 2 & 0 & 1 & 0\end{array}$ \\
\hline$\left[\begin{array}{llllll}2 & 0 & 1 & 2 & 2 & 1\end{array}\right]$ & {$\left[\begin{array}{llllll}1 & 2 & 2 & 1 & 2 & 0\end{array}\right]$} & {$\left[\begin{array}{llllll}2 & 0 & 2 & 2 & 1 & 2\end{array}\right]$} & {$\left[\begin{array}{llllll}2 & 0 & 2 & 1 & 0 & 0\end{array}\right]$} \\
\hline$\left[\begin{array}{llllll}2 & 2 & 0 & 2 & 2 & 1\end{array}\right]$ & {$\left[\begin{array}{llllll}3 & 2 & 2 & 3 & 0 & 2\end{array}\right]$} & {$\left[\begin{array}{llllll}1 & 2 & 2 & 3 & 2 & 0\end{array}\right]$} & {$\left[\begin{array}{llllll}0 & 1 & 0 & 0 & 0 & 2\end{array}\right]$} \\
\hline $\begin{array}{llllll}1 & 2 & 2 & 0 & 2 & 3\end{array}$ & $\begin{array}{llllll}2 & 2 & 2 & 1 & 2 & 0\end{array}$ & $\begin{array}{llllll}2 & 0 & 2 & 3 & 0 & 2\end{array}$ & $\begin{array}{llllll}0 & 1 & 3 & 2 & 3 & 2\end{array}$ \\
\hline$\left[\begin{array}{llllll}2 & 0 & 0 & 2 & 1 & 2\end{array}\right]$ & 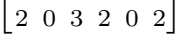 & {$\left[\begin{array}{llllll}0 & 2 & 2 & 2 & 1 & 2\end{array}\right]$} & {$\left[\begin{array}{llllll}2 & 0 & 1 & 2 & 0 & 0\end{array}\right]$} \\
\hline$\left[\begin{array}{llllll}0 & 2 & 2 & 0 & 2 & 3\end{array}\right]$ & {$\left[\begin{array}{llllll}2 & 3 & 0 & 2 & 0 & 2\end{array}\right]$} & {$\left[\begin{array}{llllll}3 & 2 & 2 & 3 & 2 & 0\end{array}\right]$} & {$\left[\begin{array}{llllll}3 & 2 & 3 & 0 & 2 & 0\end{array}\right]$} \\
\hline $\begin{array}{llllll}3 & 2 & 0 & 2 & 2 & 2\end{array}$ & $\begin{array}{lllllll}2 & 3 & 3 & 2 & 2 & 0\end{array}$ & $\begin{array}{llllll}3 & 2 & 2 & 0 & 0 & 2\end{array}$ & $\begin{array}{llllll}1 & 2 & 2 & 1 & 0 & 2\end{array}$ \\
\hline$\left[\begin{array}{llllll}2 & 0 & 1 & 2 & 2 & 1\end{array}\right]$ & {$\left[\begin{array}{llllll}2 & 2 & 2 & 0 & 1 & 2\end{array}\right]$} & {$\left[\begin{array}{llllll}2 & 2 & 0 & 2 & 2 & 3\end{array}\right]$} & {$\left[\begin{array}{llllll}3 & 2 & 0 & 0 & 2 & 2\end{array}\right]$} \\
\hline
\end{tabular}

\section{CONCLUSION}

In this paper, a new class of 4-PSK Balanced STTC for several transmit antennas has been proposed. These codes generate the points of the constellation with the same probability. It has been shown that the best STTC belong to this class. Therefore, the systematic search for good codes can be reduced to this class. A method to design the balanced codes has been described. A complete list of the best 4-state codes with 2 transmit antennas and several 16-state codes for 2 and 3 antennas have also been given. All the 4-state fully balanced STTC listed in this paper are equivalent, i.e. they have the same rank, trace and product distance. The simulation results have shown that they outperform the other 4-state STTC for 2 transmit antennas.

\section{REFERENCES}

[1] V. Tarokh, N. Seshadri, and A.R. Calderbank, "Space-time codes for high data rate wireless communication: Performance criterion and code construction," IEEE Trans. Inform. Theory, vol. 44, no. 2, pp. 744-765, March 1998.

[2] Z. Chen, J. Yuan, and B. Vucetic, "Improved space-time trellis coded modulation scheme on slow fading channels," Electron. Lett., vol. 37, no. 7, pp. 440-441, March 2001.

[3] W. Firmanto, B.S. Vucetic, and J. Yuan, "Space-time TCM with improved performance on fast fading channels," IEEE Commun. Lett., vol. 5, no. 4, pp. 154-156, April 2001.

[4] S. Baro, G. Bauch, and A. Hansmann, "Improved codes for space-time trellis-coded modulation," IEEE Commun. Lett., vol. 4, no. 1, pp. 20-22, Jan. 2000.

[5] Yi Hong and A. Guillen i Fabregas, "New Space-Time Trellis Codes for Slow Fading Channels," in Proc. IEEE VTC 2006-Spring, May 2006, vol. 3, pp. $1492-1496$.

[6] B.A. Rassool, F. Heliot, L. Revelly, M. Dohler, R. Nakhai, and H. Aghvami, "Fast search techniques for obtaining space-time trellis codes for Rayleigh fading channels and its performance in CDMA systems," in Proc. IEEE VTC 2003-Spring, April 2003, vol. 1, pp. 66-69.

[7] M. Crussiere, J.M. Auffray, and J.F. Helard, "Comparison of STTCM over slow and fast Rayleigh fading channels," in 4th IEEE Workshop on SPAWC 2003, June 2003, pp. 249-253.

[8] Z. Chen, B.S. Vucetic, J. Yuan, and Ka Leong Lo, "Space-time trellis codes for 4-PSK with three and four transmit antennas in quasi-static flat fading channels," IEEE Commun. Lett., vol. 6, no. 2, pp. 67-69, Feb. 2002.

[9] T.M.H. Ngo, G. Zaharia, S. Bougeard, and J.F. Helard, "4-PSK balanced STTC with two transmit antennas," in Proc. IEEE VTC 2007-Spring, April 2007.

[10] Yi Hong, J. Yuan, Z. Chen, and B. Vucetic, "Space-time turbo trellis codes for two, three, and four transmit antennas," IEEE Trans. on Vehicular Technology, vol. 53, no. 2, pp. 318-328, March 2004. 